\title{
CONICAL GEODESIC BICOMBINGS ON SUBSETS OF NORMED VECTOR SPACES
}

\author{
GIULIANO BASSO AND BENJAMIN MIESCH
}

\begin{abstract}
In this paper we establish existence and uniqueness results for conical geodesic bicombings on subsets of normed vector spaces. Concerning existence, we give a first example of a convex geodesic bicombing that is not consistent. Furthermore, we show that under a mild geometric assumption on the norm a conical geodesic bicombing on an open subset of a normed vector space locally consists of linear geodesics. As an application, we obtain by the use of a Cartan-Hadamard type result that if a closed convex subset $C$ of a Banach space has non-empty interior, then it admits a unique consistent conical geodesic bicombing, namely the one given by the linear segments.
\end{abstract}

\section{Introduction}

Let $(X, d)$ denote a metric space. A map $\sigma: X \times X \times[0,1] \rightarrow X$ is said to be a geodesic bicombing if the path $\sigma_{p q}(\cdot):=\sigma(p, q, \cdot)$ is a constant speed geodesic from $p$ to $q$ for all points $p, q$ in $X$, that is, we have

$$
\sigma_{p q}(0)=p, \sigma_{p q}(1)=q \text { and } d\left(\sigma_{p q}(t), \sigma_{p q}(s)\right)=|t-s| d(p, q)
$$

for all real numbers $s, t \in[0,1]$. Essentially, a geodesic bicombing distinguishes a class of geodesics of a metric space. The study of metric spaces with distinguished geodesics traces back to the influential work of H. Busemann, cf. [BP87. In this article we consider metric spaces with distinguished geodesics that satisfy the following weak, but non-coarse, global non-positive curvature condition: A geodesic bicombing $\sigma: X \times X \times[0,1] \rightarrow X$ is called conical if it satisfies the conical property

$$
d\left(\sigma_{p q}(t), \sigma_{p^{\prime} q^{\prime}}(t)\right) \leq(1-t) d\left(p, p^{\prime}\right)+t d\left(q, q^{\prime}\right)
$$

for all points $p, q, p^{\prime}, q^{\prime} \in X$ and all real numbers $t \in[0,1]$. Note that 1.1 does not imply convexity of the distance function $t \mapsto d\left(\sigma_{p q}(t), \sigma_{p^{\prime} q^{\prime}}(t)\right)$ as we will see below. The notion of a conical geodesic bicombing was coined by U. Lang in connection with injective metric spaces (also called hyperconvex metric spaces), where conical geodesic bicombings are obtained naturally, cf. [Lan13, Proposition 3.8]. Readily verified examples of metric spaces that admit conical geodesic bicombings also include convex subsets of normed vector spaces and Busemann spaces. The class of metric spaces that admit conical geodesic bicombings is by no means limited to these examples, as it follows from first principles that it is closed under ultralimits and 1-Lipschitz retractions.

Date: May 22, 2018

2010 Mathematics Subject Classification. 46B20, 46B22, 51F99, 53C22.

Keywords. Nonpositive curvature; Geodesic bicombing; Convex sets. 
Recently, classical results from the theory of CAT(0) spaces have been transferred to metric spaces that admit conical geodesic bicombings, cf. Bas17, Des16, DL16, Mie16] and Kel16]. In the past century, notions related to conical geodesic bicombings have also been considered in metric fixed point theory, most notable W-convexity mappings, cf. [Tak70, and hyperbolic spaces in the sense of S. Reich and I. Shafrir, cf. RS90. It is worth to point out that the study of metric spaces that admit conical geodesic bicombing may also lead to new results about word hyperbolic groups, as every word hyperbolic group acts geometrically on a proper, finite dimensional metric space with a unique consistent conical geodesic bicombing (the definitions are given below), cf. DL15]. The main results of this article show that the several definitions from DL15] lead to different classes.

Our first result deals with convex geodesic bicombings. From now on, we abbreviate $\mathrm{D}(X):=X \times X \times[0,1]$. A geodesic bicombing $\sigma: \mathrm{D}(X) \rightarrow X$ is convex if the map $t \mapsto d\left(\sigma_{p q}(t), \sigma_{p^{\prime} q^{\prime}}(t)\right)$ is convex on $[0,1]$ for all points $p, q, p^{\prime}, q^{\prime}$ in $X$. Note that if the underlying metric space is not uniquely geodesic, then a conical geodesic bicombing is not necessarily convex. Examples of conical geodesic bicombings that are not convex are ubiquitous; for instance, non-convex conical geodesic bicombings may be obtained via 1-Lipschitz retractions of linear geodesics, see DL15, Example 2.2] or Lemma 3.1. In [DL15, it is shown that metric spaces of finite combinatorial dimension in the sense of Dress, cf. Dre84, possess at most one convex geodesic bicombing. If it exists, this unique convex geodesic bicombing, say $\sigma: \mathrm{D}(X) \rightarrow X$, has the property that it is consistent, that is, we have for all points $p, q$ in $X$ that $\operatorname{im}\left(\sigma_{p^{\prime} q^{\prime}}\right) \subset \operatorname{im}\left(\sigma_{p q}\right)$ whenever $p^{\prime}=\sigma_{p q}(s)$ and $q^{\prime}=\sigma_{p q}(t)$ with $0 \leq s \leq t \leq 1$. Clearly, every consistent conical geodesic bicombing is convex. In Section 2 we show that the converse does not hold by proving the subsequent theorem.

Theorem 1.1. There is a compact metric space that admits a convex geodesic bicombing which is not consistent.

Although there is a non-consistent convex geodesic bicombing on the space considered in Section 2, this space also admits a consistent convex geodesic bicombing. We suspect that this is a general phenomenon.

Question 1.2. Let $(X, d)$ be a proper metric space with a convex geodesic bicombing. Does $X$ also admit a consistent convex geodesic bicombing?

The seemingly more general question if every proper metric space with a conical geodesic bicombing admits a consistent conical geodesic bicombing is in fact equivalent to Question 1.2, as every proper metric space with a conical geodesic bicombing also admits a convex geodesic bicombing, cf. [DL15, Theorem 1.1].

A geodesic bicombing $\sigma: \mathrm{D}(X) \rightarrow X$ is called reversible if $\sigma_{p q}(t)=\sigma_{q p}(1-t)$ for all points $p, q$ in $X$ and all $t \in[0,1]$. It is possible to modify our non-consistent convex geodesic bicombing from Theorem 1.1 in order to obtain an example of a non-reversible convex geodesic bicombing, see Proposition 2.5

In [Bas17, a barycentric construction has been employed to obtain fixed point results for metric spaces that admit conical geodesic bicombings. This barycentric construction motivated the following definition: A geodesic bicombing $\sigma: \mathrm{D}(X) \rightarrow$ $X$ has the midpoint property if $\sigma_{p q}\left(\frac{1}{2}\right)=\sigma_{q p}\left(\frac{1}{2}\right)$ for all points $p, q$ in $X$. It seems natural to ask if every conical geodesic bicombing that has the midpoint property is automatically reversible. We show that this is not the case, as we construct 
in Section 3 a non-reversible conical geodesic bicombing which has the midpoint property. We conclude Section 3 with the following proposition.

Proposition 1.3. Let $(X, d)$ be a complete metric space with a conical geodesic bicombing $\sigma$. Then $X$ also admits a reversible conical geodesic bicombing.

This generalizes the result for proper metric spaces established in Des16, Proposition 1.2].

It is a direct consequence of a result of S. Gähler and G. Murphy that the only conical geodesic bicombing on a normed vector space is the one that consists of the linear geodesics, cf. GM81, Theorem 1]. With a mild geometric assumption on the norm, we show in Section 4 that already a conical geodesic bicombing on an open subset of a normed vector space locally consists of linear geodesics. More generally, we get the following result:

Theorem 1.4. Let $(V,\|\cdot\|)$ be a normed vector space such that its closed unit ball is the closed convex hull of its extreme points. Suppose that $A \subset V$ is a subset of $V$ that admits a conical geodesic bicombing $\sigma: \mathrm{D}(A) \rightarrow A$ and let $p_{0} \in A$ be a point. If $r \geq 0$ is a real number such that the closed ball $B_{2 r}\left(p_{0}\right)$ is contained in $A$, then we have that $\sigma(p, q, t)=(1-t) p+t q$ for all points $p, q \in B_{r}\left(p_{0}\right)$ and all real numbers $t \in[0,1]$.

We do not know if Theorem 1.4 remains true if we drop the assumption of the normed vector space $(V,\|\cdot\|)$ having the property that its closed unit ball is the closed convex hull of its extreme points. But how common is this property?

By invoking the Banach-Alaoğlu theorem and the Kreln-Mil'man theorem it is possible to show that the closed unit ball of a dual Banach space is the closed convex hull of its extreme points. Consequently, we obtain in particular that Theorem 1.4 is valid in every reflexive Banach space. Moreover, using a classification result, due to L. Nachbin, D. Goodner, and J. Kelley, cf. [Kel52, and a result of D. Goodner, cf. Goo50, Theorem 6.4], it is readily verified that Theorem 1.4 also holds for every injective Banach space.

Note that the classical Mazur-Ulam Theorem is a direct consequence of Theorem 1.4, as every isometric isomorphism between two normed vector spaces extends to an isometric isomorphism between their linear injective hulls, which by the above satisfy the assumptions of Theorem 1.4 .

In [Mie16, the second named author generalized the classical Cartan-Hadamard Theorem to metric spaces that locally admit a consistent convex geodesic bicombing. With Theorem 1.4 at hand, it is possible to use this generalized CartanHadamard Theorem to obtain the following uniqueness result.

Theorem 1.5. Let $(E,\|\cdot\|)$ be a Banach space such that its closed unit ball is the closed convex hull of its extreme points. Suppose that $C \subset E$ is a closed convex subset of $E$ with non-empty interior. If $\sigma: \mathrm{D}(C) \rightarrow C$ is a consistent conical geodesic bicombing, then it follows that $\sigma(p, q, t)=(1-t) p+t q$ for all points $p, q$ in $C$ and all real numbers $t \in[0,1]$.

The proof of Theorem 1.5 is given in Section 5 In Example 4.4 we construct two distinct consistent conical geodesic bicombings on a closed convex subset $B \subset$ $L^{1}([0,1])$ with empty interior. As it is possible to consider $B$ as a subset of the 
injective hull of $L^{1}([0,1])$, it follows that the assumption in Theorem 1.5 of $C$ having non-empty interior is necessary.

Due to Theorems 1.4 and 1.5 it appears that the geometry of a convex subset $C$ with non-empty interior is very restricted in the sense that it is difficult to construct a conical geodesic bicombing on $C$ that is not given by the linear geodesics. In this perspective, we deem that a negative answer to the following question would result in an interesting geometric construction.

Question 1.6. Let $C \subset E$ be a convex subset of a Banach space $(E,\|\cdot\|)$. Suppose that $C$ has non-empty interior. Is it true that $C$ admits only one conical geodesic bicombing?

\section{A non-consistent convex geodesic bicombing}

The goal of this section is to construct a convex geodesic bicombing that is not consistent and therefore establish Theorem 1.1. To this end, we consider the following norm on $\mathbb{R}^{2}$ :

$$
\|(x, y)\|:=\max \left\{|x|, \frac{\sqrt{2}}{2}\|(x, y)\|_{2}\right\}
$$

where $\|(x, y)\|_{2}=\sqrt{x^{2}+y^{2}}$ is the Euclidean norm. Observe that $\|(x, y)\|=|x|$ if and only if $|y| \leq|x|$. Now define

$$
X:=\left\{(x, y) \in \mathbb{R}^{2}:-3 \leq x \leq 3,0 \leq y \leq \frac{1}{32} \max \left\{0,1-x^{2}\right\}\right\}
$$

and equip $X$ with the metric $d$ induced by $\|\cdot\|$, see Figure 1 .

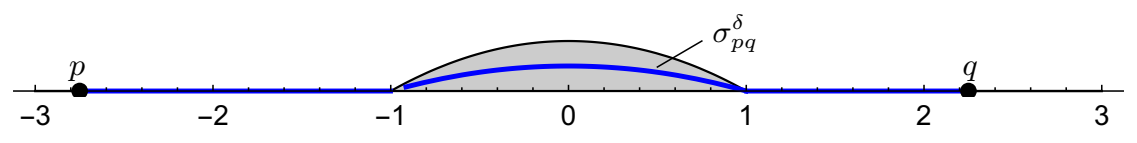

Figure 1. The metric space $X$ with a geodesic $\sigma_{p q}^{\delta}$.

The space $X$ naturally splits into three pieces, namely $X=X_{-} \cup X_{0} \cup X_{+}$with

$$
\begin{aligned}
X_{-} & :=[-3,-1] \times\{0\}, \\
X_{0} & :=\left\{(x, y) \in \mathbb{R}^{2}:-1<x<1,0 \leq y \leq \frac{1}{32}\left(1-x^{2}\right)\right\}, \\
X_{+} & :=[1,3] \times\{0\} .
\end{aligned}
$$

Definition 2.1. For $\delta \in\left[0, \frac{1}{64}\right]$ we define a geodesic bicombing $\sigma^{\delta}: \mathrm{D}(X) \rightarrow X$ as follows. Generally, we take $\sigma_{p q}^{\delta}$ to be the geodesic from $p$ to $q$ which is linear inside $X_{0}$, but if both endpoints lie on the antennas $X_{-}, X_{+}$we slightly modify it, see Figure 1. In more details $\sigma^{\delta}$ is defined as follows:

For $p:=\left(p_{x}, p_{y}\right), q:=\left(q_{x}, q_{y}\right) \in X$ with $p_{x} \leq q_{x}$ let

$$
\sigma_{p q}^{\delta}(t):=\left(x_{p q}(t), y_{p q}(t)\right)
$$


with

$$
\begin{aligned}
x_{p q}(t) & :=p_{x}+t\left(q_{x}-p_{x}\right), \\
y_{p q}(t): & := \begin{cases}\delta \max \left\{q_{x}-p_{x}-4,0\right\} \max \left\{0,\left(1-x_{p q}(t)^{2}\right)\right\}, & \text { for } p \in X_{-}, q \in X_{+}, \\
\max \left\{0, \frac{q_{y}}{q_{x}+1}\left(x_{p q}(t)+1\right)\right\}, & \text { for } p \in X_{-}, q \in X_{0}, \\
\max \left\{0, \frac{p_{y}}{p_{x}-1}\left(x_{p q}(t)-1\right)\right\}, & \text { for } p \in X_{0}, q \in X_{+}, \\
p_{y}+t\left(q_{y}-p_{y}\right), & \text { for } p, q \in X_{0}, \\
0, & \text { otherwise. }\end{cases}
\end{aligned}
$$

and

$$
\sigma_{q p}^{\delta}(t):=\sigma_{p q}^{\delta}(1-t) .
$$

Proposition 2.2. For $\delta \in\left(0, \frac{1}{64}\right]$, the map $\sigma^{\delta}$ is a reversible convex geodesic bicombing which is not consistent.

Remark 2.3. Observe that for $\delta=0$ the geodesic bicombing $\sigma^{\delta}$ coincides with the piecewise linear bicombing which is the unique consistent conical geodesic bicombing on $X$ by Theorem 1.5. Hence we have a family of non-consistent convex geodesic bicombings $\sigma^{\delta}$ converging to the unique consistent convex geodesic bicombing $\sigma^{0}$.

Alternatively, we can modify the geodesics leading from $X_{-}$to $X_{+}$so that we lose the reversibility.

Definition 2.4. Define $\tilde{\sigma}^{\delta}: \mathrm{D}(X) \rightarrow X$ by

$$
\tilde{\sigma}_{p q}^{\delta}(t)=\sigma_{p q}^{\delta}(t)
$$

except for $p \in X_{+}, q \in X_{-}$let

$$
\tilde{\sigma}_{p q}^{\delta}=\left(x_{p q}(t), 0\right) .
$$

Proposition 2.5. For $\delta \in\left(0, \frac{1}{64}\right]$, the map $\tilde{\sigma}^{\delta}$ is a convex geodesic bicombing which is neither reversible nor consistent.

The proofs of Propositions 2.2 and 2.5 are given in the appendix. Let us first show that we have defined geodesic bicombings.

Lemma 2.6. For $\delta \in\left[0, \frac{1}{64}\right]$, the maps $\sigma^{\delta}$ and $\tilde{\sigma}^{\delta}$ are geodesic bicombings.

Proof. The linear case is clear. For the piecewise linear case observe that if $p \in X_{-}$, $q \in X_{0}$ (and similarly in all other cases) we have that the slope $m$ of $\sigma_{p q}^{\delta}$ satisfies

$$
m=\frac{q_{y}}{q_{x}+1} \leq \frac{\frac{1}{32}\left(1-q_{x}^{2}\right)}{1+q_{x}}=\frac{1}{32}\left(1-q_{x}\right) \leq \frac{1}{16} \leq 1
$$

and therefore

$$
d\left(\sigma_{p q}^{\delta}(s), \sigma_{p q}^{\delta}(t)\right)=\left|x_{p q}(s)-x_{p q}(t)\right|=|s-t|\left|q_{x}-p_{x}\right|=|s-t| d(p, q) .
$$

Finally, let $p \in X_{-}, q \in X_{+}$. For $x, x^{\prime} \in[-1,1]$ we have

$$
\begin{aligned}
& \mid \delta\left(q_{x}-p_{x}-4\right)\left(1-x^{2}\right)-\delta\left(q_{x}-p_{x}-4\right)\left(1-x^{2}\right) \mid \\
& \leq \delta\left|q_{x}-p_{x}-4\right| \cdot\left|x+x^{\prime}\right| \cdot\left|x-x^{\prime}\right| \leq \frac{1}{16}\left|x-x^{\prime}\right|
\end{aligned}
$$

and hence $d\left(\sigma_{p q}^{\delta}(s), \sigma_{p q}^{\delta}(t)\right)=\left|x_{p q}(s)-x_{p q}(t)\right|$ as before. 
It is immediate that both geodesic bicombings are non-consistent. Furthermore, $\sigma^{\delta}$ is reversible and $\tilde{\sigma}^{\delta}$ is not. Hence, it remains to prove convexity.

Given $p, q, p^{\prime}, q^{\prime} \in X$ we need to show that the function $f(t):=d\left(\sigma_{p q}^{\delta}(t), \sigma_{p^{\prime} q^{\prime}}^{\delta}(t)\right)$ is convex on $[0,1]$. To this end, we use the following characterization of convexity; see Lemma 3.5 in [LY10.

Lemma 2.7. A continuous function $f:[0,1] \rightarrow \mathbb{R}$ is convex if and only if for every $t \in(0,1)$ there is some $\tau_{0}>0$ such that for all $\tau \in\left[0, \tau_{0}\right]$ we have

$$
2 f(t) \leq f(t-\tau)+f(t+\tau)
$$

Now, let $t \in(0,1)$. In the situation when $d\left(\sigma_{p q}^{\delta}(t), \sigma_{p^{\prime} q^{\prime}}^{\delta}(t)\right)=\left|x_{p q}(t)-x_{p^{\prime} q^{\prime}}(t)\right|$, we have

$$
\begin{aligned}
2 d\left(\sigma_{p q}^{\delta}(t), \sigma_{p^{\prime} q^{\prime}}^{\delta}(t)\right) & =2\left|x_{p q}(t)-x_{p^{\prime} q^{\prime}}(t)\right| \\
& \leq\left|x_{p q}(t-\tau)-x_{p^{\prime} q^{\prime}}(t-\tau)\right|+\left|x_{p q}(t+\tau)-x_{p^{\prime} q^{\prime}}(t+\tau)\right| \\
& \leq d\left(\sigma_{p q}^{\delta}(t-\tau), \sigma_{p^{\prime} q^{\prime}}^{\delta}(t-\tau)\right)+d\left(\sigma_{p q}^{\delta}(t+\tau), \sigma_{p^{\prime} q^{\prime}}^{\delta}(t+\tau)\right),
\end{aligned}
$$

as $t \mapsto\left|x_{p q}(t)-x_{p^{\prime} q^{\prime}}(t)\right|$ is convex. Therefore, it remains to check

$$
\begin{aligned}
& 2\left\|\sigma_{p q}^{\delta}(t)-\sigma_{p^{\prime} q^{\prime}}^{\delta}(t)\right\|_{2} \\
& \leq\left\|\sigma_{p q}^{\delta}(t-\tau)-\sigma_{p^{\prime} q^{\prime}}^{\delta}(t-\tau)\right\|_{2}+\left\|\sigma_{p q}^{\delta}(t+\tau)-\sigma_{p^{\prime} q^{\prime}}^{\delta}(t+\tau)\right\|_{2}
\end{aligned}
$$

for $\tau>0$ small, whenever $d\left(\sigma_{p q}^{\delta}(t), \sigma_{p^{\prime} q^{\prime}}^{\delta}(t)\right)=\frac{\sqrt{2}}{2}\left\|\sigma_{p q}^{\delta}(t)-\sigma_{p^{\prime} q^{\prime}}^{\delta}(t)\right\|_{2}$, that is, $\left|x_{p q}(t)-x_{p^{\prime} q^{\prime}}(t)\right| \leq\left|y_{p q}(t)-y_{p^{\prime} q^{\prime}}(t)\right|$.

In this case, the main reason for convexity is that the modification in the $y$ direction is controlled by the speed difference in the $x$-direction. To illustrate this, let us consider $\sigma_{p q}^{\delta}$ and $\sigma_{p^{\prime} q^{\prime}}^{\delta}$ for $p=(-3,0), q=(3,0), p^{\prime}=(-2,0), q^{\prime}=(2,0)$. Note that for $t \in\left[\frac{1}{3}, \frac{2}{3}\right], \sigma_{p q}^{\delta}(t)$ lies on the (concave) parabola $2 \delta\left(1-x^{2}\right)$ while $\sigma_{p^{\prime} q^{\prime}}^{\delta}$ describes a linear segment on the $x$-axis. However, e.g. for $t=\frac{1}{2}$, we have

$$
\begin{aligned}
\left\|\sigma_{p q}\left(\frac{1}{2} \pm \tau\right)-\sigma_{p^{\prime} q^{\prime}}\left(\frac{1}{2} \pm \tau\right)\right\|_{2}^{2} & =(3 \tau-2 \tau)^{2}+4 \delta^{2}\left(1-9 \tau^{2}\right)^{2} \\
& =4 \delta^{2}+\left(1-72 \delta^{2}\right) \tau^{2}+324 \delta^{2} \tau^{4} \\
& \geq 4 \delta^{2}=\|\left(\sigma_{p q}\left(\frac{1}{2}\right)-\sigma_{p^{\prime} q^{\prime}}\left(\frac{1}{2}\right) \|_{2}^{2}\right.
\end{aligned}
$$

for $\delta \in\left(0, \frac{1}{\sqrt{72}}\right]$ and consequently, 2.1) follows.

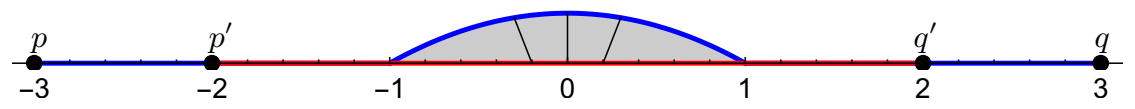

Figure 2. The function $t \mapsto d\left(\sigma_{p q}^{\delta}(t), \sigma_{p^{\prime} q^{\prime}}^{\delta}(t)\right)$ is convex.

A similar calculation can also be carried out for all other pairings of geodesics of the bicombing. To this end, we shall distinguish several cases. This is done in the appendix, where detailed proofs of Propositions 2.2 and 2.5 are given. 


\section{Reversibility of conical geodesic bicombings}

In the first part of this section we construct a non-reversible conical geodesic bicombing. Afterwards, we modify this non-reversible conical geodesic bicombing to satisfy the midpoint property. Finally, we prove Proposition 1.3 .

Consider $\mathbb{R}^{2}$ equipped with the maximum norm $\|\cdot\|_{\infty}$ and let $s: \mathbb{R}^{2} \rightarrow \mathbb{R}^{2}$ denote the map given by $(x, y) \mapsto(x,-y)$. We define

$$
\begin{aligned}
& X_{1}:=\left\{(x, y) \in \mathbb{R}^{2}: x \in[-2,1] \text { and }|x|-1 \leq y \leq|| x|-1|\right\}, \\
& A_{1}:=\left\{(x, y) \in \mathbb{R}^{2}:|x+1| \leq y \leq 1\right\} .
\end{aligned}
$$

and $X_{2}:=s\left(X_{1}\right), A_{2}:=s\left(A_{1}\right)$. The set $X_{1} \cup X_{2}$ is depicted in Figure 3. It is readily verified that the map $f: X_{2} \rightarrow X_{1}$ given by

$$
(x, y) \mapsto \begin{cases}(x, y), & \text { if } x \in[-1,1], \\ s(x, y), & \text { if } x \in[-2,-1]\end{cases}
$$

is an isometry. Let $\bar{f}: X_{1} \cup X_{2} \rightarrow X_{1}$ be the map that is equal to $\operatorname{Id}_{X_{1}}$ on $X_{1}$ and equal to $f$ on $X_{2}$. Observe that the map $\bar{f}$ is 1-Lipschitz. We set $Y_{k}:=X_{k} \cup A_{k}$ for $k \in\{1,2\}$.

Further, we define the map $\pi: Y_{1} \cup Y_{2} \rightarrow X_{1} \cup X_{2}$ through the assignment

$$
(x, y) \mapsto(x, \operatorname{sgn}(y) \min \{|y|,|| x|-1|\}) .
$$

Observe that $\pi$ is a 1-Lipschitz retraction that maps $Y_{k}$ to $X_{k}$ for each $k \in\{1,2\}$. Let $\lambda: \mathrm{D}\left(\mathbb{R}^{2}\right) \rightarrow \mathbb{R}^{2}$ be the conical geodesic bicombing on $\mathbb{R}^{2}$ that is given by the linear geodesics.

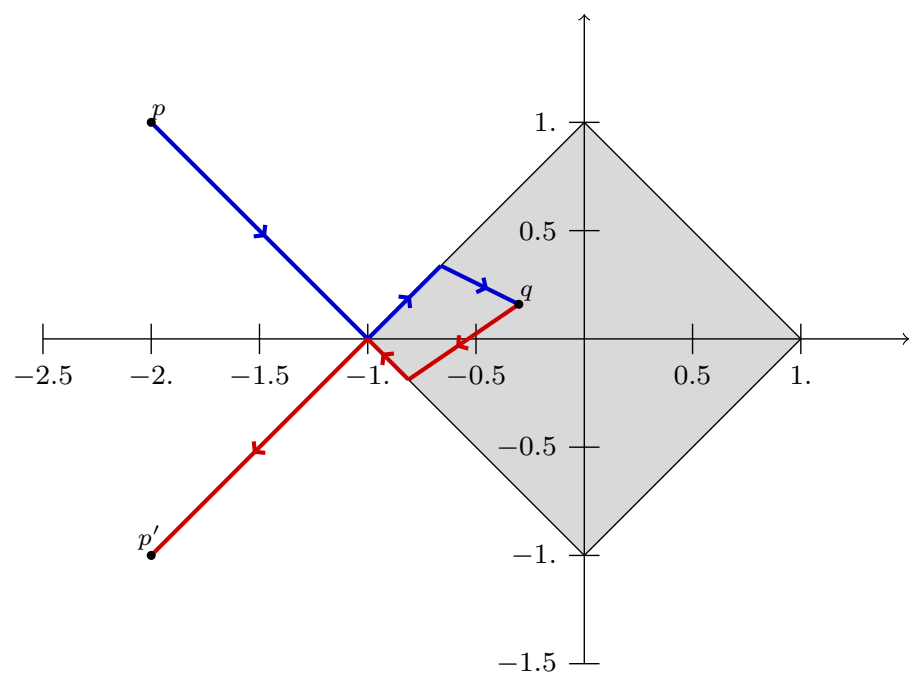

Figure 3. The blue line corresponds to $\sigma_{p q}$ and the red line corresponds to the image of $\sigma_{q p}$ under the isometry $f^{-1}$. 
Lemma 3.1. The map $\sigma: \mathrm{D}\left(X_{1}\right) \rightarrow X_{1}$ given by

$$
(p, q, t) \mapsto \begin{cases}\pi \circ \lambda(p, q, t), & \text { if } p_{x} \leq q_{x}, \\ f \circ \pi \circ \lambda\left(f^{-1}(p), f^{-1}(q), t\right), & \text { if } q_{x} \leq p_{x} .\end{cases}
$$

is a non-reversible conical geodesic bicombing on $\left(X_{1},\|\cdot\|_{\infty}\right)$.

Proof. Observe that both maps

$$
\sigma^{(1)}:=\pi \circ \lambda \text { and } \sigma^{(2)}:=f \circ \pi \circ \lambda \circ\left(f^{-1} \times f^{-1} \times \operatorname{Id}_{[0,1]}\right)
$$

define conical geodesic bicombings on $X_{1}$. Thus, it follows that $\sigma: \mathrm{D}\left(X_{1}\right) \rightarrow X_{1}$ is a geodesic bicombing.

In the following we show that $\sigma$ is conical. Let $p, q, p^{\prime}, q^{\prime} \in X_{1}$ be points. As both maps $\sigma^{(1)}$ and $\sigma^{(2)}$ are conical geodesic bicombings on $X_{1}$ with $\sigma_{p q}^{(1)}=\sigma_{p q}^{(2)}$ if $p_{x}, q_{x} \leq-1$ or $p_{x}, q_{x} \geq-1$, it remains to check inequality (1.1) if $\left(p_{x}, q_{x}^{\prime} \leq-1\right.$ and $\left.q_{x}, p_{x}^{\prime} \geq-1\right)$ or $\left(p_{x}^{\prime}, q_{x} \leq-1\right.$ and $\left.q_{x}^{\prime}, p_{x} \geq-1\right)$.

Now, suppose that $p_{x}, q_{x}^{\prime} \leq-1$ and $q_{x}, p_{x}^{\prime} \geq-1$. The other case is treated analogously. Since the map $\bar{f} \circ \pi$ is 1-Lipschitz, we compute

$$
\begin{aligned}
\left\|\sigma_{p q}(t)-\sigma_{p^{\prime} q^{\prime}}(t)\right\|_{\infty} & =\left\|\bar{f} \circ \pi \circ \lambda(p, q, t)-\bar{f} \circ \pi \circ \lambda\left(f^{-1}\left(p^{\prime}\right), f^{-1}\left(q^{\prime}\right), t\right)\right\|_{\infty} \\
& \leq(1-t)\left\|p-f^{-1}\left(p^{\prime}\right)\right\|_{\infty}+t\left\|q-f^{-1}\left(q^{\prime}\right)\right\|_{\infty}
\end{aligned}
$$

for all $t \in[0,1]$. By our assumptions on the points $p, q, p^{\prime}, q^{\prime}$, it follows that

$$
\begin{aligned}
& \left\|p-f^{-1}\left(p^{\prime}\right)\right\|_{\infty}=\left\|p-p^{\prime}\right\|_{\infty}, \\
& \left\|q-f^{-1}\left(q^{\prime}\right)\right\|_{\infty}=\left\|f^{-1}(q)-f^{-1}\left(q^{\prime}\right)\right\|_{\infty}=\left\|q-q^{\prime}\right\|_{\infty} .
\end{aligned}
$$

Hence, by putting everything together, we obtain that $\sigma$ is a conical geodesic bicombing on $X_{1}$. By construction, it follows that $\sigma$ is non-reversible; see Figure 3 .

Now, we use the conical geodesic bicombing from Lemma 3.1 to construct a non-reversible conical geodesic bicombing that has the midpoint property.

Lemma 3.2. Let $\sigma: \mathrm{D}\left(X_{1}\right) \rightarrow X_{1}$ denote the map from Lemma 3.1. The map $\tau: \mathrm{D}\left(X_{1}\right) \rightarrow X_{1}$ given by the assignment

$$
(p, q, t) \mapsto \begin{cases}\sigma\left(p, \frac{1}{2}\left(\sigma\left(p, q, \frac{1}{2}\right)+\sigma\left(q, p, \frac{1}{2}\right)\right), 2 t\right), & \text { if } t \in\left[0, \frac{1}{2}\right], \\ \sigma\left(\frac{1}{2}\left(\sigma\left(p, q, \frac{1}{2}\right)+\sigma\left(q, p, \frac{1}{2}\right)\right), q, 2 t-1\right), & \text { if } t \in\left[\frac{1}{2}, 1\right],\end{cases}
$$

is a conical geodesic bicombing on $\left(X_{1},\|\cdot\|_{\infty}\right)$ that has the midpoint property but is not reversible.

Proof. It is readily verified that $\tau$ is a conical geodesic bicombing with the midpoint property. To see that $\tau$ is non-reversible, take for instance $p:=\left(-\frac{3}{2}, \frac{1}{2}\right), q:=$ $\left(0, \frac{1}{2}\right)$ and observe that $\tau\left(p, q, \frac{5}{12}\right)=\left(-\frac{7}{8}, \frac{1}{8}\right) \neq\left(-\frac{7}{8}, \frac{1}{48}\right)=\tau\left(q, p, \frac{7}{12}\right)$; compare Figure 4.

To prove Proposition 1.3 we need the following midpoint construction:

Lemma 3.3. Let $(X, d)$ be a complete metric space. If $\sigma: \mathrm{D}(X) \rightarrow X$ is a conical geodesic bicombing, then there is a midpoint map $m: X \times X \rightarrow X$ with the following properties: for all points $x, y, \bar{x}, \bar{y} \in X$ we have

(i) $m(x, y)=m(y, x)$, 


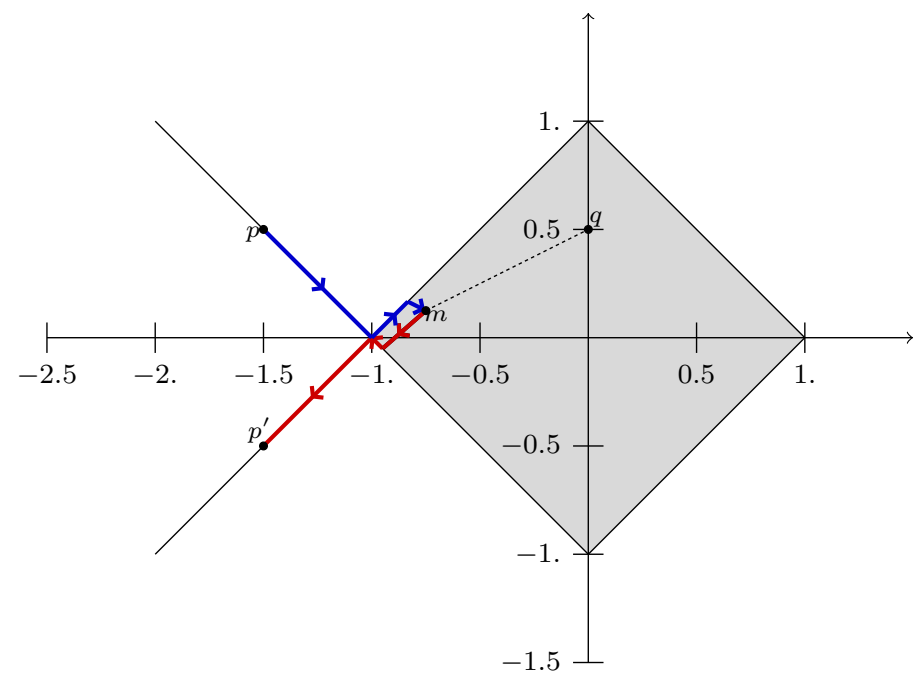

Figure 4 . The blue line corresponds to $\left.\tau_{p q}\right|_{\left[0, \frac{1}{2}\right]}$ and the red line corresponds to the image of $\left.\tau_{q p}\right|_{\left[\frac{1}{2}, 1\right]}$ under the isometry $f^{-1}$. The point $m$ is equal to $\frac{1}{2}\left(\sigma_{p q}\left(\frac{1}{2}\right)+\sigma_{q p}\left(\frac{1}{2}\right)\right)$.

(ii) $d(x, m(x, y))=d(y, m(x, y))=\frac{1}{2} d(x, y)$,

(iii) $d(m(x, y), m(\bar{x}, \bar{y})) \leq \frac{1}{2} d(x, \bar{x})+\frac{1}{2} d(y, \bar{y})$.

Proof. Let $x, y \in X$. Set $x_{0}:=x, y_{0}:=y$ and define recursively $x_{n+1}:=$ $\sigma\left(x_{n}, y_{n}, \frac{1}{2}\right), y_{n+1}:=\sigma\left(y_{n}, x_{n}, \frac{1}{2}\right)$. We have

$$
\begin{aligned}
d\left(x_{n+1}, y_{n+1}\right) & =d\left(\sigma\left(x_{n}, y_{n}, \frac{1}{2}\right), y_{n+1}\right) \\
& \leq \frac{1}{2} d\left(x_{n}, y_{n+1}\right)+\frac{1}{2} d\left(y_{n}, y_{n+1}\right)=\frac{1}{2} d\left(x_{n}, y_{n}\right)
\end{aligned}
$$

and therefore $d\left(x_{n}, y_{n}\right) \leq \frac{1}{2^{n}} d(x, y), d\left(x_{n}, x_{n-1}\right) \leq \frac{1}{2^{n}} d(x, y)$. Hence the sequences $\left(x_{n}\right)_{n \geq 0},\left(y_{n}\right)_{n \geq 0}$ are Cauchy and converge to some common limit point $m(x, y)$.

By the construction, we clearly have $(i)$. To prove $(i i)$ we claim that $d\left(x, x_{n}\right), d\left(x, y_{n}\right), d\left(y, x_{n}\right), d\left(y, y_{n}\right) \leq \frac{1}{2} d(x, y)$ for all $n \geq 1$. This follows by induction since $d\left(x, x_{n+1}\right) \leq \frac{1}{2} d\left(x, x_{n}\right)+\frac{1}{2} d\left(x, y_{n}\right) \leq \frac{1}{2} d(x, y)$ and similar for all other distances. It remains to show (iii). If we repeat the construction for $\bar{x}, \bar{y} \in X$ we get some sequences $\left(\bar{x}_{n}\right)_{n \geq 0},\left(\bar{y}_{n}\right)_{n \geq 0}$ with limit point $m(\bar{x}, \bar{y})$. We now prove by induction that $d\left(x_{n}, \bar{x}_{n}\right), d\left(y_{n}, \bar{y}_{n}\right) \leq \frac{1}{2} d(x, \bar{x})+\frac{1}{2} d(y, \bar{y})$ for all $n \geq 1$. Indeed, we have

$$
\begin{aligned}
d\left(x_{n+1}, \bar{x}_{n+1}\right) & =d\left(\sigma\left(x_{n}, y_{n}, \frac{1}{2}\right), \sigma\left(\bar{x}_{n}, \bar{y}_{n}, \frac{1}{2}\right)\right) \\
& \leq \frac{1}{2} d\left(x_{n}, \bar{x}_{n}\right)+\frac{1}{2} d\left(y_{n}, \bar{y}_{n}\right) \leq \frac{1}{2} d(x, \bar{x})+\frac{1}{2} d(y, \bar{y}),
\end{aligned}
$$

and similarly $d\left(y_{n+1}, \bar{y}_{n+1}\right) \leq \frac{1}{2} d(x, \bar{x})+\frac{1}{2} d(y, \bar{y})$. Hence, statement (iii) follows by taking the limit $n \rightarrow+\infty$.

Proof of Proposition 1.3. We define a new bicombing $\tau: \mathrm{D}(X) \rightarrow X$ by

$$
\tau(x, y, t):=m(\sigma(x, y, t), \sigma(y, x, 1-t)) .
$$


For two points $x, y \in X$ this defines a geodesic from $x$ to $y$, since for $s, t \in[0,1]$ we have

$$
\begin{aligned}
d(\tau(x, y, t), \tau(x, y, s)) & =d(m(\sigma(x, y, t), \sigma(y, x, 1-t)), m(\sigma(x, y, s), \sigma(y, x, 1-s)) \\
& \leq \frac{1}{2} d(\sigma(x, y, t), \sigma(x, y, s))+\frac{1}{2} d(\sigma(y, x, 1-t), \sigma(y, x, 1-s)) \\
& =|s-t| d(x, y) .
\end{aligned}
$$

Moreover, the conical inequality holds, as we have

$$
\begin{aligned}
d(\tau(x, y, t), \tau(\bar{x}, \bar{y}, t)) & =d(m(\sigma(x, y, t), \sigma(y, x, 1-t)), m(\sigma(\bar{x}, \bar{y}, t), \sigma(\bar{y}, \bar{x}, 1-t))) \\
& \leq \frac{1}{2} d(\sigma(x, y, t), \sigma(\bar{x}, \bar{y}, t))+\frac{1}{2} d(\sigma(y, x, 1-t), \sigma(\bar{y}, \bar{x}, 1-t)) \\
& \leq(1-t) d(x, \bar{x})+t d(y, \bar{y}),
\end{aligned}
$$

for all $x, y, \bar{x}, \bar{y} \in X$ and $t \in[0,1]$.

\section{Local behavior of conical geodesic bicombings}

Let $(V,\|\cdot\|)$ be a normed vector space, let $p_{0} \in V$ be a point and let $r \geq 0$ be a real number. We set

$$
\begin{aligned}
U_{r}\left(p_{0}\right) & :=\left\{z \in V:\left\|p_{0}-z\right\|<r\right\}, \\
B_{r}\left(p_{0}\right) & :=\left\{z \in V:\left\|p_{0}-z\right\| \leq r\right\}, \\
S_{r}\left(p_{0}\right) & :=\left\{z \in V:\left\|p_{0}-z\right\|=r\right\} .
\end{aligned}
$$

To ease notation, we abbreviate $B_{r}:=B_{r}(0)$ and $S_{r}:=S_{r}(0)$. The goal of this section is to establish the following rigidity result.

Theorem 4.1. Let $(V,\|\cdot\|)$ be a normed vector space. Suppose that $A \subset V$ is a subset of $V$ that admits a conical geodesic bicombing $\sigma: \mathrm{D}(A) \rightarrow A$ and let $p, q$ be points of $A$. If there are points $e_{1}, \ldots, e_{n} \in B_{1}$ that are extreme points of $B_{1}$ and a tuple $\left(\lambda_{1}, \ldots, \lambda_{n}\right) \in[0,1]^{n}$ with $\sum_{k=1}^{n} \lambda_{k}=1$ such that

$$
\begin{aligned}
& \frac{p-q}{2}=\frac{\|p-q\|}{2} \sum_{k=1}^{n} \lambda_{k} e_{k} \text { and } \\
& \frac{p+q}{2}+\frac{\|p-q\|}{2}\left\{\sum_{k=1}^{n}(-1)^{\varepsilon_{k}} \lambda_{k} e_{k}:\left(\varepsilon_{1}, \ldots, \varepsilon_{n}\right) \in\{0,1\}^{n}\right\} \subset A,
\end{aligned}
$$

then it follows that $\sigma(p, q, t)=(1-t) p+t q$ for all $t \in[0,1]$.

Theorem 1.4 then is a direct consequence.

Proof of Theorem 1.4. Let $p, q \in B_{r}\left(p_{0}\right)$ be two points. As $\frac{p+q}{2} \in B_{r}\left(p_{0}\right)$ and $\frac{\|p-q\|}{2} \leq r$, the ball $B_{\frac{\|p-q\|}{2}}\left(\frac{p+q}{2}\right)$ is contained in $A$. Hence, since the unit ball of $V$ is the closed convex hull of its extreme points, it follows that $\sigma(p, q, t)=(1-t) p+t q$ for all $t \in[0,1]$ by Theorem 4.1 and a simple limit argument.

We will derive Theorem 4.1 via induction on the number of extreme points. For this induction, we need some preparatory lemmas and definitions.

We define the map $\lambda: \mathrm{D}(V) \rightarrow V$ via the assignment

$$
(p, q, t) \mapsto(1-t) p+t q .
$$


It is readily verified that $\lambda$ is a conical geodesic bicombing. Let $t \in[0,1]$ be a real number and let $p, q$ be points in $V$. We define

$$
M^{(t)}(p, q):=\{z \in V:\|z-p\|=t\|p-q\|,\|z-q\|=(1-t)\|p-q\|\} .
$$

Clearly, $\sigma(p, q, t) \in M^{(t)}(p, q)$ for every geodesic bicombing $\sigma$. Thus, if $M^{(t)}(p, q)$ is a singleton, then $\sigma(p, q, t)=\lambda(p, q, t)$. The first lemma of this section gives a sufficient condition for the set $M^{(t)}(p, q)$ to be a singleton.

Lemma 4.2. Let $(V,\|\cdot\|)$ be a normed vector space and let $p \in V$ be a point. If $p$ is an extreme point of $B_{\|p\|}$, then $M^{(t)}(p,-p)=\{(1-2 t) p\}$ for all $t \in[0,1]$.

Proof. By construction, we have

$$
M^{(t)}(p,-p)=\left(S_{2 t\|p\|}+p\right) \cap\left(S_{(1-t) 2\|p\|}-p\right) ;
$$

hence,

$$
\frac{1}{2 t}\left(p-M^{(t)}(p,-p)\right)=S_{\|p\|} \cap\left(\frac{1}{t} p-\frac{1-t}{t} S_{\|p\|}\right),
$$

provided that $t \in(0,1]$. For each $t \in(0,1]$ we define the map $E^{(t)}: V \rightarrow \mathcal{P}(V)$ via the assignment

$$
p \mapsto S_{\|p\|} \cap\left(\frac{1}{t} p-\frac{1-t}{t} S_{\|p\|}\right) .
$$

Note that $\mathcal{P}(V)$ denotes the power set of $V$. By the use of the identity 4.3 $M^{(t)}(p,-p)=\{(1-2 t) p\}$ if and only if $E^{(t)}(p)=\{p\}$. Thus, we are left to show that if $p$ is an extreme point of $B_{\|p\|}$, then $E^{(t)}(p)=\{p\}$ for all $t \in(0,1)$. We argue by contraposition. Suppose that there is a real number $t \in(0,1)$ and a point $p^{\prime} \in E^{(t)}(p)$ with $p^{\prime} \neq p$. As $p^{\prime} \in E^{(t)}(p)$, it follows that $p^{\prime} \in S_{\|p\|}$ and that there is a point $q \in S_{\|p\|}$ such that $p^{\prime}=\frac{1}{t} p-\frac{1-t}{t} q$. Observe that $q \neq p$ and

$$
(1-t) q+t p^{\prime}=(1-t) q+t\left(\frac{1}{t} p-\frac{1-t}{t} q\right)=p .
$$

Hence the point $p$ is not extreme in $B_{\|p\|}$, as desired. By putting everything together, the lemma follows.

Lemma 4.2 will serve as base case for the induction in the proof of Theorem 4.1 . The subsequent lemma is the key component for the inductive step in the proof of Theorem 4.1.

Lemma 4.3. Let $(V,\|\cdot\|)$ be a normed vector space and let $A \subset V$ be a subset that admits a conical geodesic bicombing $\sigma: \mathrm{D}(A) \rightarrow A$. Let $p$ be a point in $A$ such that $-p \in A$. If there is a point $z$ in $V$ such that the points $2 z-p$ and $p-2 z$ are contained in $A$ and such that $\sigma(p, p-2 z, \cdot)=\lambda(p, p-2 z, \cdot)$ and $\sigma(2 z-p,-p, \cdot)=$ $\lambda(2 z-p,-p, \cdot)$, then we have that

$$
\sigma(p,-p, t) \in\left((1-2 t) z+M^{(t)}(p-z, z-p)\right) .
$$

for all real numbers $t \in[0,1]$.

Proof. Let $t \in[0,1]$ be a real number. Using that $\sigma$ is conical, we compute

$$
\begin{aligned}
\|\sigma(p,-p, t)-\lambda(p, p-2 z, t)\| & \leq 2 t\|p-z\| \\
\|\sigma(p,-p, t)-\lambda(2 z-p,-p, t)\| & \leq 2(1-t)\|p-z\| .
\end{aligned}
$$


Note that $\|\lambda(p, p-2 z, t)-\lambda(2 z-p,-p, t)\|=2\|p-z\|$. Therefore, it follows that

$$
\sigma(p,-p, t) \in M^{(t)}(\lambda(p, p-2 z, t), \lambda(2 z-p,-p, t)) .
$$

It is readily verified that $M^{(t)}(u+h, v+h)=h+M^{(t)}(u, v)$ for all $t$ in $[0,1]$ and $u, v, h \in V$. Consequently, we obtain that

$$
M^{(t)}(\lambda(p, p-2 z, t), \lambda(2 z-p,-p, t))=(1-2 t) z+M^{(t)}(p-z, z-p) .
$$

Thus, the lemma follows.

Suppose that $A$ is a subset of a normed vector space $(V,\|\cdot\|)$ and assume that $A$ admits a conical geodesic bicombing $\sigma: \mathrm{D}(A) \rightarrow A$. The translation $T_{z}: A \rightarrow T_{z}(A)$ about the vector $z \in V$ given by the assignment $x \mapsto x+z$ is an isometry and the $\operatorname{map}\left(T_{z}\right)_{*} \sigma: \mathrm{D}\left(T_{z}(A)\right) \rightarrow T_{z}(A)$ given by

$$
(x, y, t) \mapsto T_{z}\left(\sigma\left(T_{-z}(x), T_{-z}(y), t\right)\right)
$$

is a conical geodesic bicombing on $T_{z}(A)$. Now, we have everything on hand to prove Theorem 4.1 .

Proof of Theorem 4.1. We proceed by induction on $n \geq 1$. If $n=1$, then Lemma 4.2 tells us that

$$
\left(T_{-\frac{p+q}{2}}\right)_{*} \sigma\left(\frac{p-q}{2},-\frac{p-q}{2}, t\right)=(1-2 t) \frac{p-q}{2}
$$

for all $t \in[0,1]$. Thus, we obtain that $\sigma(p, q, t)=(1-t) p+t q$ for all $t \in[0,1]$.

Suppose now that $n>1$ and that the statement holds for $n-1$. We may assume that $\lambda_{1} \in(0,1)$. We define $\left(\lambda_{1}^{\prime}, \ldots, \lambda_{n-1}^{\prime}\right):=\frac{1}{1-\lambda_{1}}\left(\lambda_{2}, \ldots, \lambda_{n}\right)$ and $\left(e_{1}^{\prime}, \ldots, e_{n-1}^{\prime}\right):=\left(e_{2}, \ldots, e_{n}\right)$. Observe that

$$
\sum_{k=1}^{n} \lambda_{k} e_{k}=\lambda_{1} e_{1}+\left(1-\lambda_{1}\right) \sum_{k=1}^{n-1} \lambda_{k}^{\prime} e_{k}^{\prime}
$$

Further, note that

$$
\left\|\sum_{k=1}^{n-1} \lambda_{k}^{\prime} e_{k}^{\prime}\right\|=1 \text {, as otherwise 4.5 implies }\left\|\sum_{k=1}^{n} \lambda_{k} e_{k}\right\|<1,
$$

which is not possible due to 4.1. We abbreviate $r:=\frac{\|p-q\|}{2}$ and we set

$$
z:=r\left(1-\lambda_{1}\right) \sum_{k=1}^{n-1} \lambda_{k}^{\prime} e_{k}^{\prime}, \quad \quad p^{\prime}:=\frac{p-q}{2}, \quad q^{\prime}:=p^{\prime}-2 z .
$$

Note that

$$
\frac{p^{\prime}-q^{\prime}}{2}=r\left(1-\lambda_{1}\right) \sum_{k=1}^{n-1} \lambda_{k}^{\prime} e_{k}^{\prime} .
$$

Hence, by the use of 4.6 it follows that

$$
\frac{\left\|p^{\prime}-q^{\prime}\right\|}{2}=r\left(1-\lambda_{1}\right)
$$

We have that

$$
\frac{p^{\prime}+q^{\prime}}{2}=\frac{p-q}{2}-z \stackrel{4.1}{=} r \sum_{k=1}^{n} \lambda_{k} e_{k}-r\left(1-\lambda_{1}\right) \sum_{k=1}^{n-1} \lambda_{k}^{\prime} e_{k}^{\prime} \stackrel{4.5)}{=} r \lambda_{1} e_{1}
$$


and therefore

$$
\begin{aligned}
& \frac{p^{\prime}+q^{\prime}}{2}+\frac{\left\|p^{\prime}-q^{\prime}\right\|}{2}\left\{\sum_{k=1}^{n-1}(-1)^{\varepsilon_{k}} \lambda_{k}^{\prime} e_{k}^{\prime}:\left(\varepsilon_{1}, \ldots, \varepsilon_{n-1}\right) \in\{0,1\}^{n-1}\right\} \\
& \stackrel{4.7]}{-} r\left\{\lambda_{1} e_{1}+\sum_{k=2}^{n}(-1)^{\varepsilon_{k}} \lambda_{k} e_{k}:\left(\varepsilon_{2} \ldots, \varepsilon_{n}\right) \in\{0,1\}^{n-1}\right\} \stackrel{4.2]}{C} T_{-\frac{p+q}{2}}(A) .
\end{aligned}
$$

Thus, we can apply the induction hypothesis to $p^{\prime}, q^{\prime} \in T_{-\frac{p+q}{2}}(A)$ and obtain that

$$
\left(T_{-\frac{p+q}{2}}\right)_{*} \sigma\left(p^{\prime}, p^{\prime}-2 z, \cdot\right)=\lambda\left(p^{\prime}, p^{\prime}-2 z, \cdot\right) .
$$

Similarly, we obtain

$$
\left(T_{-\frac{p+q}{2}}\right)_{*} \sigma\left(2 z-p^{\prime},-p^{\prime}, \cdot\right)=\lambda\left(2 z-p^{\prime},-p^{\prime}, \cdot\right) .
$$

Now, by the use of Lemma 4.3 it follows that

$$
\left(T_{-\frac{p+q}{2}}\right)_{*} \sigma\left(p^{\prime},-p^{\prime}, t\right) \in\left((1-2 t) z+M^{(t)}\left(p^{\prime}-z, z-p^{\prime}\right)\right)
$$

for all real numbers $t \in[0,1]$; consequently, we get

$$
\left(T_{-\frac{p+q}{2}}\right)_{*} \sigma\left(p^{\prime},-p^{\prime}, t\right)=(1-2 t) p^{\prime}
$$

since $p^{\prime}-z=r \lambda_{1} e_{1}$ is an extreme point in $B_{r \lambda_{1}}$ and thus we can use Lemma 4.2 to deduce that $M^{(t)}\left(p^{\prime}-z, z-p^{\prime}\right)=\left\{(1-2 t)\left(p^{\prime}-z\right)\right\}$. Hence, we have

$$
\sigma(p, q, t)=\left(T_{-\frac{p+q}{2}}\right)_{*} \sigma\left(p^{\prime},-p^{\prime}, t\right)+\frac{p+q}{2}=(1-t) p+t q,
$$

as desired.

We conclude this section with an example of a closed convex subset of a Banach space that admits two distinct consistent conical geodesic bicombings.

Example 4.4. We define the set

$A:=\{f:[0,1] \rightarrow[0,1]: f(0)=0, f(1)=1, f$ is continuous and strictly increasing $\}$.

We claim that the metric space $\left(A,\|\cdot\|_{1}\right)$ admits two distinct consistent conical geodesic bicombings. Clearly, as $A$ is convex, the map $\lambda: \mathrm{D}(A) \rightarrow A$ given by $(f, g, t) \mapsto(1-t) f+t g$ is a consistent conical geodesic bicombing on $\left(A,\|\cdot\|_{1}\right)$. Let $\varphi: A \rightarrow A$ denote the map given by $f \mapsto f^{-1}$. The map $\varphi$ is an isometry of $\left(A,\|\cdot\|_{1}\right)$. This is a simple consequence of the identity

$$
\|f-g\|_{1}=\operatorname{vol}_{2}\left(\left\{(x, y) \in[0,1]^{2}: \min \{f(x), g(x)\} \leq y \leq \max \{f(x), g(x)\}\right\}\right)
$$

which holds true for all $f, g \in A$ and where $\operatorname{vol}_{2}$ denotes the two dimensional Lebesgue measure.

Let $\tau: \mathrm{D}(A) \rightarrow A$ be the map where each map $\tau_{f g}(\cdot)$ is given by the horizontal interpolation of the functions $f, g \in A$, that is, the map $\tau$ is given by the assignment $(f, g, t) \mapsto \varphi((1-t) \varphi(f)+t \varphi(g))$. As the map $\varphi$ is an isometry, it follows that $\tau$ is a consistent conical geodesic bicombing. Indeed, it holds that $\tau=\varphi_{*} \lambda$, here we use the notation introduced in (4.4). Furthermore, if $f(x):=\sqrt{x}$ and $g(x):=x$, then we have that the map $\tau(f, g, t):[0,1] \rightarrow[0,1]$ is given by

$$
x \mapsto \frac{-t+\sqrt{4(1-t) x+t^{2}}}{2(1-t)}
$$


for all $t \in[0,1]$, which is distinct from $\lambda(f, g, t)=(1-t) f+t g$ for all $t \in(0,1)$. Hence, the metric space $\left(A,\|\cdot\|_{1}\right)$ admits two distinct consistent conical geodesic bicombings. Let $B$ denote the closure of $A \subset L^{1}([0,1])$. Note that $\lambda$ and $\tau$ extend naturally to consistent conical geodesic bicombings on $B$. Hence, we have found a closed convex subset of a Banach space that admits two distinct consistent conical geodesic bicombings. It is readily verified that $B$ has empty interior.

\section{Proof of Theorem 1.5}

Before we start with the proof of Theorem 1.5, we recall some notions from Mie16. Let $(X, d)$ be a metric space, let $p \in X$ be a point and let $r>0$ be a real number. We set $U_{r}(p):=\{q \in X: d(p, q)<r\}$. Let $U \subset \mathrm{D}(X)$ be a subset. A map $\sigma: U \rightarrow X$ is a convex local geodesic bicombing if for every point $p \in X$ there is a real number $r_{p}>0$ such that

$$
U=\bigcup_{p \in X} \mathrm{D}\left(U_{r_{p}}(p)\right)
$$

and if the restriction $\left.\sigma\right|_{\mathrm{D}\left(U_{r_{p}}(p)\right)}: \mathrm{D}\left(U_{r_{p}}(p)\right) \rightarrow X$ is a consistent conical geodesic bicombing for each point $p \in X$. Furthermore, we say that a geodesic $c:[0,1] \rightarrow X$ is consistent with the convex local geodesic bicombing $\sigma$ if for each choice of real numbers $0 \leq s_{1} \leq s_{2} \leq 1$ with $\left(c\left(s_{1}\right), c\left(s_{2}\right)\right) \in U_{r_{p}}(p) \times U_{r_{p}}(p)$ for some point $p \in X$, it holds

for all $t \in[0,1]$.

$$
c\left((1-t) s_{1}+t s_{2}\right)=\sigma\left(c\left(s_{1}\right), c\left(s_{2}\right), t\right)
$$

Consistent geodesics are uniquely determined by the local geodesic bicombing, compare [Mie16, Theorem 1.1] and the proof thereof:

Theorem 5.1. Let $X$ be a complete, simply-connected metric space with a convex local geodesic bicombing $\sigma$. If we equip $X$ with the length metric, then for every two points $p, q \in X$ there is a unique geodesic from $p$ to $q$ which is consistent with $\sigma$ and the collection of all such geodesics is a convex geodesic bicombing.

With Theorem 5.1 on hand it is possible to derive Theorem 1.5 by the use of Theorem 1.4 .

Proof of Theorem 1.5. Let int $(C)$ denote the interior of $C$ and let $p, q$ be two points in $\operatorname{int}(C)$. We abbreviate

$$
[p, q]:=\{(1-t) p+t q: t \in[0,1]\} .
$$

As int $(C)$ is convex, we have that $[p, q] \subset \operatorname{int}(C)$. For each point $z \in C$ we set

$$
r_{z}:= \begin{cases}\min \{\|z-w\|: w \in[p, q]\} & \text { if } z \in C \backslash \operatorname{int}(C) \\ \frac{1}{2} \inf \{\|z-w\|: w \in C \backslash \operatorname{int}(C)\} & \text { if } z \in \operatorname{int}(C) .\end{cases}
$$

Note that $r_{z}>0$ for all points $z \in C$ and we have that $U_{r_{z}}(z) \cap[p, q]=\varnothing$ if $z \in$ $C \backslash \operatorname{int}(C)$. Further, for every point $z \in \operatorname{int}(C)$ it follows that $B_{2 r_{z}}(z) \subset C$; thus, we may invoke Theorem 1.4 to deduce that if $z \in \operatorname{int}(C)$, then $\sigma_{z_{1} z_{2}}(t)=(1-t) z_{1}+t z_{2}$ for all points $z_{1}, z_{2} \in B_{r_{z}}(z)$ and all real numbers $t \in[0,1]$. We define

$$
U:=\bigcup_{z \in C} \mathrm{D}\left(U_{r_{z}}(z)\right) .
$$


Note that the map $\sigma^{\text {loc }}:=\left.\sigma\right|_{U}$ defines a convex local bicombing on $C$. The geodesic $\sigma_{p q}(\cdot)$ and the linear geodesic from $p$ to $q$ are both consistent with the local bicombing $\sigma^{\text {loc }}$. Hence, by Theorem 5.1 we conclude that $\sigma_{p q}(\cdot)$ must be equal to the linear geodesic from $p$ to $q$, that is, we have $\sigma_{p q}(t)=(1-t) p+t q$ for all real numbers $t \in[0,1]$.

Now, suppose that $p, q \in C$. As $C$ is convex, it is well-known that $C=\overline{\operatorname{int}(C)}$, cf. [AB06, Lemma 5.28]. Let $\left(p_{k}\right)_{k \geq 1},\left(q_{k}\right)_{k \geq 1} \subset \operatorname{int}(C)$ be two sequences such that $p_{k} \rightarrow p$ and $q_{k} \rightarrow q$ with $k \rightarrow+\infty$. It is readily verified that $\sigma_{p_{k} q_{k}}(\cdot) \rightarrow \sigma_{p q}(\cdot)$ with $k \rightarrow+\infty$, since $\sigma$ is a conical geodesic bicombing. As a result, we obtain that the geodesic $\sigma_{p q}(\cdot)$ is equal to the linear geodesic from $p$ to $q$, as desired.

\section{A Proofs of Propositions 2.2 and 2.5}

For the sake of completeness, we add here the remaining, quite technical details in the proofs of Propositions 2.2 and 2.5 which were stated in Section 2

Proof of Proposition 2.2. As we have already mentioned in Section 2, the geodesic bicombing $\sigma^{\delta}$ is non-consistent and reversible. Moreover, in the situation when $d\left(\sigma_{p q}^{\delta}(t), \sigma_{p^{\prime} q^{\prime}}^{\delta}(t)\right)=\left|x_{p q}(t)-x_{p^{\prime} q^{\prime}}(t)\right|$ we have

$$
2 d\left(\sigma_{p q}^{\delta}(t), \sigma_{p^{\prime} q^{\prime}}^{\delta}(t)\right) \leq d\left(\sigma_{p q}^{\delta}(t-\tau), \sigma_{p^{\prime} q^{\prime}}^{\delta}(t-\tau)\right)+d\left(\sigma_{p q}^{\delta}(t+\tau), \sigma_{p^{\prime} q^{\prime}}^{\delta}(t+\tau)\right) .
$$

Therefore, let us check

$$
\begin{aligned}
& 2\left\|\sigma_{p q}^{\delta}(t)-\sigma_{p^{\prime} q^{\prime}}^{\delta}(t)\right\|_{2} \\
& \leq\left\|\sigma_{p q}^{\delta}(t-\tau)-\sigma_{p^{\prime} q^{\prime}}^{\delta}(t-\tau)\right\|_{2}+\left\|\sigma_{p q}^{\delta}(t+\tau)-\sigma_{p^{\prime} q^{\prime}}^{\delta}(t+\tau)\right\|_{2}
\end{aligned}
$$

for $\tau>0$ small, whenever $d\left(\sigma_{p q}^{\delta}(t), \sigma_{p^{\prime} q^{\prime}}^{\delta}(t)\right)=\frac{\sqrt{2}}{2}\left\|\sigma_{p q}^{\delta}(t)-\sigma_{p^{\prime} q^{\prime}}^{\delta}(t)\right\|_{2}$, that is, $\left|x_{p q}(t)-x_{p^{\prime} q^{\prime}}(t)\right| \leq\left|y_{p q}(t)-y_{p^{\prime} q^{\prime}}(t)\right|$.

Observe that for $x \in[-3,-1] \cup[1,3]$ and $\left(x^{\prime}, y^{\prime}\right) \in X$ we have that $d\left((x, 0),\left(x^{\prime}, y^{\prime}\right)\right)=\left|x-x^{\prime}\right|$ and therefore we always have $d\left(\sigma_{p q}^{\delta}(t), \sigma_{p^{\prime} q^{\prime}}^{\delta}(t)\right)=$ $\left|x_{p q}(t)-x_{p^{\prime} q^{\prime}}(t)\right|$ if $x_{p q}(t) \notin(-1,1)$. Hence, we only need to consider points that satsify $x_{p q}(t), x_{p^{\prime} q^{\prime}}(t) \in(-1,1)$.

First, if both $\sigma_{p q}^{\delta}, \sigma_{p^{\prime} q^{\prime}}^{\delta}$ are (piece-wise) linear, then locally they are linear geodesics inside a normed vector space and hence $d\left(\sigma_{p q}(t), \sigma_{p^{\prime} q^{\prime}}(t)\right)=\| \sigma_{p q}(t)-$ $\sigma_{p^{\prime} q^{\prime}}(t) \|$ is locally convex, thus convex.

Let us now assume that $\sigma_{p q}^{\delta}$ is not linear, i.e. $p \in X_{-}, q \in X_{+}, l:=d(p, q) \geq 4$. We look at the different options for $\sigma_{p^{\prime} q^{\prime}}^{\delta}$ separately. But before doing so, let us first fix some notation. We define $p_{0}:=\sigma_{p q}(t), p_{ \pm}:=\sigma_{p q}(t \pm \tau), p_{*}=\left(x_{*}, y_{*}\right)$ $(* \in\{0,+,-\}), D:=\delta(l-4), \varepsilon:=\tau l$ and accordingly for $\sigma_{p^{\prime} q^{\prime}}^{\delta}$. We then get $y_{0}=D\left(1-x_{0}^{2}\right), x_{ \pm}=x_{0} \pm \varepsilon$ and $y_{ \pm}=D\left(1-\left(x_{0} \pm \varepsilon\right)^{2}\right)$.

In each case, we need to consider the situation where $x_{0}, x_{0}^{\prime} \in(-1,1)$ and $\left|x_{0}-x_{0}^{\prime}\right| \leq\left|y_{0}-y_{0}^{\prime}\right|$.

Case 1. $p^{\prime} \in X_{\mp}, q^{\prime} \in X_{ \pm}$and $l^{\prime}:=d\left(p^{\prime}, q^{\prime}\right) \in[4, l]$.

As above we have $y_{0}^{\prime}=D^{\prime}\left(1-x_{0}^{\prime 2}\right), x_{ \pm}^{\prime}=x_{0}^{\prime} \pm \varepsilon^{\prime}, y_{ \pm}^{\prime}=D^{\prime}\left(1-\left(x_{0}^{\prime} \pm \varepsilon^{\prime}\right)^{2}\right)$ and with $\lambda:=\frac{l^{\prime}}{l}$ we get $\varepsilon^{\prime}=\lambda \varepsilon$. 
We claim that

$$
2\left\|p_{0}-p_{0}^{\prime}\right\|_{2} \leq\left\|p_{-}-p_{-}^{\prime}\right\|_{2}+\left\|p_{+}-p_{+}^{\prime}\right\|_{2}
$$

for $\varepsilon>0$ (i.e. $\tau>0$ ) small enough.

First note that

$$
\begin{aligned}
& \left\|p_{-}-p_{-}^{\prime}\right\|_{2}^{2}=\left\|p_{0}-p_{0}^{\prime}\right\|_{2}^{2}-2\left(x_{0}-x_{0}^{\prime}\right)(1-\lambda) \varepsilon+(1-\lambda)^{2} \varepsilon^{2}+2\left(y_{0}-y_{0}^{\prime}\right) a \varepsilon+a^{2} \varepsilon^{2}, \\
& \left\|p_{+}-p_{+}^{\prime}\right\|_{2}^{2}=\left\|p_{0}-p_{0}^{\prime}\right\|_{2}^{2}+2\left(x_{0}-x_{0}^{\prime}\right)(1-\lambda) \varepsilon+(1-\lambda)^{2} \varepsilon^{2}+2\left(y_{0}-y_{0}^{\prime}\right) b \varepsilon+b^{2} \varepsilon^{2},
\end{aligned}
$$

for

$$
\begin{aligned}
a & :=2\left(x_{0} D-\lambda x_{0}^{\prime} D^{\prime}\right)-\left(D-\lambda^{2} D^{\prime}\right) \varepsilon, \\
b & :=-2\left(x_{0} D-\lambda x_{0}^{\prime} D^{\prime}\right)-\left(D-\lambda^{2} D^{\prime}\right) \varepsilon,
\end{aligned}
$$

with $a+b=-2\left(D-\lambda^{2} D^{\prime}\right) \varepsilon, a-b=4\left(x_{0} D-\lambda x_{0}^{\prime} D^{\prime}\right)$ and either $a b=\left(D-\lambda^{2} D^{\prime}\right)^{2} \varepsilon^{2}$ or $a b<0$ for $\varepsilon$ small. In the following, we assume $a b<0$. The other case is similar. Moreover, we have

$$
\begin{aligned}
& \left\|p_{-}-p_{-}^{\prime}\right\|_{2}^{2} \cdot\left\|p_{+}-p_{+}^{\prime}\right\|_{2}^{2}=\left\|p_{0}-p_{0}^{\prime}\right\|_{2}^{4} \\
& \quad+\left(4 a b\left(y-y^{\prime}\right)^{2}-4\left(x_{0}-x_{0}^{\prime}\right)^{2}(1-\lambda)^{2}+4\left(x_{0}-x_{0}^{\prime}\right)(1-\lambda)\left(y_{0}-y_{0}^{\prime}\right)(a-b)\right. \\
& \left.\quad+\left(2(1-\lambda)^{2}+a^{2}+b^{2}-4\left(y_{0}-y_{0}^{\prime}\right)\left(D-\lambda^{2} D^{\prime}\right)\right) \cdot\left\|p_{0}-p_{0}^{\prime}\right\|_{2}^{2}\right) \varepsilon^{2}+\mathcal{O}\left(\varepsilon^{3}\right)
\end{aligned}
$$

and with $\sqrt{u+t}=\sqrt{u}+\frac{t}{2 \sqrt{u}}+\mathcal{O}\left(t^{2}\right)$ and $u=\left\|p_{0}-p_{0}^{\prime}\right\|_{2}^{4}$ it follows

$$
\begin{aligned}
2 & \sqrt{\left\|p_{-}-p_{-}^{\prime}\right\|_{2}^{2} \cdot\left\|p_{+}-p_{+}^{\prime}\right\|_{2}^{2}} \\
\geq & 2\left\|p_{0}-p_{0}^{\prime}\right\|_{2}^{2}+\left(2(1-\lambda)^{2}+a^{2}+b^{2}+4 a b-4\left(y_{0}-y_{0}^{\prime}\right)\left(D-\lambda^{2} D^{\prime}\right)\right. \\
& \left.+\frac{4\left(x_{0}-x_{0}^{\prime}\right)\left(y_{0}-y_{0}^{\prime}\right)(1-\lambda)(a-b)-4\left(x_{0}-x_{0}^{\prime}\right)^{2}(1-\lambda)^{2}}{\left(x_{0}-x_{0}^{\prime}\right)^{2}+\left(y_{0}-y_{0}^{\prime}\right)^{2}}\right) \varepsilon^{2}+\mathcal{O}\left(\varepsilon^{3}\right) .
\end{aligned}
$$

We therefore get

$$
\begin{aligned}
\left(\| p_{-}-\right. & \left.p_{-}^{\prime}\left\|_{2}+\right\| p_{+}-p_{+}^{\prime} \|_{2}\right)^{2} \\
= & \left\|p_{-}-p_{-}^{\prime}\right\|_{2}^{2}+\left\|p_{+}-p_{+}^{\prime}\right\|_{2}^{2}+2 \sqrt{\left\|p_{-}-p_{-}^{\prime}\right\|_{2}^{2} \cdot\left\|p_{+}-p_{+}^{\prime}\right\|_{2}^{2}} \\
\geq & 4\left\|p_{0}-p_{0}^{\prime}\right\|_{2}^{2}+\left(4(1-\lambda)^{2}+2(a+b)^{2}-8\left(y_{0}-y_{0}^{\prime}\right)\left(D-\lambda^{2} D^{\prime}\right)\right. \\
& \left.+\frac{4\left(x_{0}-x_{0}^{\prime}\right)\left(y_{0}-y_{0}^{\prime}\right)(1-\lambda)(a-b)-4\left(x_{0}-x_{0}^{\prime}\right)^{2}(1-\lambda)^{2}}{\left(x_{0}-x_{0}^{\prime}\right)^{2}+\left(y_{0}-y_{0}^{\prime}\right)^{2}}\right) \varepsilon^{2}+\mathcal{O}\left(\varepsilon^{3}\right) \\
= & 4\left\|p_{0}-p_{0}^{\prime}\right\|_{2}^{2}+C \varepsilon^{2}+\mathcal{O}\left(\varepsilon^{3}\right) \\
\geq & 4\left\|p_{0}-p_{0}^{\prime}\right\|_{2}^{2},
\end{aligned}
$$

for $\varepsilon>0$ small enough, provided that

$$
\begin{aligned}
C= & 4(1-\lambda)^{2}-8\left(y_{0}-y_{0}^{\prime}\right)\left(D-\lambda^{2} D^{\prime}\right) \\
& +\frac{16\left(x_{0}-x_{0}^{\prime}\right)\left(y_{0}-y_{0}^{\prime}\right)(1-\lambda)\left(x_{0} D-\lambda x_{0}^{\prime} D^{\prime}\right)-4\left(x_{0}-x_{0}^{\prime}\right)^{2}(1-\lambda)^{2}}{\left(x_{0}-x_{0}^{\prime}\right)^{2}+\left(y_{0}-y_{0}^{\prime}\right)^{2}}>0 .
\end{aligned}
$$


Observe that $a+b=\mathcal{O}(\varepsilon)$. Thus, we are left to show that $C>0$. Assuming $y_{0}>y_{0}^{\prime}$, we have

$$
\begin{aligned}
y_{0}-y_{0}^{\prime} & =D\left(1-x_{0}^{2}\right)-D^{\prime}\left(1-x_{0}^{2}\right)=\left(D-D^{\prime}\right)\left(1-x_{0}^{2}\right)+D^{\prime}\left(x_{0}^{2}-x_{0}^{2}\right) \\
& \leq \delta\left(l-l^{\prime}\right)+\delta\left(l^{\prime}-4\right)\left(x_{0}^{\prime}+x_{0}\right)\left(x_{0}^{\prime}-x_{0}\right) \leq \delta\left(l-l^{\prime}\right)+4 \delta\left(y_{0}-y_{0}^{\prime}\right)
\end{aligned}
$$

and therefore

$$
\left|y_{0}-y_{0}^{\prime}\right| \leq \frac{\delta}{1-4 \delta}\left(l-l^{\prime}\right)
$$

Moreover,

$$
\begin{aligned}
\left|D-\lambda^{2} D^{\prime}\right| l^{2} & =\delta\left(l^{3}-4 l-l^{\prime 3}+4 l^{\prime}\right) \\
& =\delta\left(l-l^{\prime}\right)\left(l^{2}+l l^{\prime}+l^{\prime 2}-4\left(l+l^{\prime}\right)\right) \leq 60 \delta\left(l-l^{\prime}\right), \\
\left|x_{0} D-\lambda x_{0}^{\prime} D^{\prime}\right| l & \leq\left|x_{0}\right|\left(D-\lambda D^{\prime}\right) l+\left|x_{0}-x_{0}^{\prime}\right| D^{\prime} l^{\prime} \\
& \leq \delta\left(l-l^{\prime}\right)\left(l+l^{\prime}-4\right)+12 \delta\left|y_{0}-y_{0}^{\prime}\right| \leq\left(8 \delta+\frac{12 \delta^{2}}{1-4 \delta}\right)\left(l-l^{\prime}\right) .
\end{aligned}
$$

Hence, we finally get

$$
\begin{aligned}
C l^{2}\left\|p_{0}-p_{0}^{\prime}\right\|_{2}^{2}= & 4\left(l-l^{\prime}\right)^{2}\left(y_{0}-y_{0}^{\prime}\right)^{2} \\
& -8\left(y_{0}-y_{0}^{\prime}\right)\left(D-\lambda^{2} D^{\prime}\right) l^{2}\left(\left(x_{0}-x_{0}^{\prime}\right)^{2}+\left(y_{0}-y_{0}^{\prime}\right)^{2}\right) \\
& +16\left(x_{0}-x_{0}^{\prime}\right)\left(y_{0}-y_{0}^{\prime}\right)\left(l-l^{\prime}\right)\left(x_{0} D-\lambda x_{0}^{\prime} D^{\prime}\right) l \\
\geq & \left(4-\frac{960 \delta^{2}}{1-4 \delta}-128 \delta-\frac{192 \delta^{2}}{1-4 \delta}\right)\left(l-l^{\prime}\right)^{2}\left(y_{0}-y_{0}^{\prime}\right)^{2} \\
= & \left(\frac{4-144 \delta-640 \delta^{2}}{1-4 \delta}\right)\left(l-l^{\prime}\right)^{2}\left(y_{0}-y_{0}^{\prime}\right)^{2}>0
\end{aligned}
$$

for $\delta<\frac{1}{40}$. This is especially true for $\delta \leq \frac{1}{64}$.

Case 2. $\sigma_{p^{\prime} q^{\prime}}$ piece-wise linear with $p^{\prime} \notin X_{0}$ or $q^{\prime} \notin X_{0}$.

Let $m$ be the slope of $\sigma_{p^{\prime} q^{\prime}}$ at $p_{0}^{\prime}$. If $p^{\prime} \in X_{-}$and $q^{\prime} \in X_{0}$, then we have

$$
m=\frac{q_{y}^{\prime}}{q_{x}^{\prime}+1} \leq \frac{\frac{1}{32}\left(1-{q_{x}^{\prime}}^{2}\right)}{1+q_{x}}=\frac{1}{32}\left(1-q_{x}^{\prime}\right) \leq \frac{1}{32}\left(4-l^{\prime}\right) \leq \frac{1}{32}\left(l-l^{\prime}\right),
$$

and similarly we also get in all other cases $|m| \leq \frac{1}{32}\left(l-l^{\prime}\right)$ and especially $|m| \leq 1$.

Moreover, we have $l \in[4,6], l^{\prime} \in[0,4]$ and for $\varepsilon^{\prime}=\tau l^{\prime}, \lambda=\frac{s^{\prime}}{s}$ we get $x_{ \pm}^{\prime}=x_{0}^{\prime} \pm \varepsilon^{\prime}$, $y_{ \pm}^{\prime}=y_{0}^{\prime} \pm m \varepsilon^{\prime}$ and $\varepsilon^{\prime}=\lambda \varepsilon$.

We can proceed as before with

$$
\begin{aligned}
& a=\lambda m+2 D x_{0}-D \varepsilon, \\
& b=-\lambda m-2 D x_{0}-D \varepsilon,
\end{aligned}
$$

and we finally get the constant

$$
\begin{aligned}
C= & 4(1-\lambda)^{2}-8\left(y_{0}-y_{0}^{\prime}\right) D \\
+ & \frac{8\left(x_{0}-x_{0}^{\prime}\right)\left(y_{0}-y_{0}^{\prime}\right)(1-\lambda)\left(\lambda m+2 D x_{0}\right)-4\left(x_{0}-x_{0}^{\prime}\right)^{2}(1-\lambda)^{2}}{\left(x_{0}-x_{0}^{\prime}\right)^{2}+\left(y_{0}-y_{0}^{\prime}\right)^{2}} .
\end{aligned}
$$


With

$$
\begin{aligned}
D & =\delta(l-4) \leq \delta\left(l-l^{\prime}\right), \\
y_{0}-y_{0}^{\prime} & \leq D\left(1-x_{0}^{2}\right) \leq D \leq \delta\left(l-l^{\prime}\right)
\end{aligned}
$$

it follows

$$
\begin{aligned}
C l^{2}\left\|p_{0}-p_{0}^{\prime}\right\|_{2}^{2}= & 4\left(l-l^{\prime}\right)^{2}\left(y_{0}-y_{0}^{\prime}\right)^{2} \\
& -8\left(y_{0}-y_{0}^{\prime}\right) D l^{2}\left(\left(x_{0}-x_{0}^{\prime}\right)^{2}+\left(y_{0}-y_{0}^{\prime}\right)^{2}\right) \\
& +8\left(x_{0}-x_{0}^{\prime}\right)\left(y_{0}-y_{0}^{\prime}\right)\left(l-l^{\prime}\right)\left(m l^{\prime}+2 D x_{0} l\right) \\
\geq & \left(4-576 \delta^{2}-1-96 \delta\right)\left(l-l^{\prime}\right)^{2}\left(y_{0}-y_{0}^{\prime}\right)^{2} \\
= & \left(3-96 \delta-576 \delta^{2}\right)\left(l-l^{\prime}\right)^{2}\left(y_{0}-y_{0}^{\prime}\right)^{2}>0
\end{aligned}
$$

for $\delta<0.026$.

Case 3. $\sigma_{p^{\prime} q^{\prime}}$ linear with $p^{\prime}, q^{\prime} \in X_{0}$.

Let $m$ again denote the slope of $\sigma_{p^{\prime} q^{\prime}}$. We distinguish two subcases.

(a) If $|m| \leq 1$, we have $l \in[4,6], l^{\prime} \in[0,2]$ and

$$
\left|m l^{\prime}\right|=\frac{\left|q_{y}^{\prime}-p_{y}^{\prime}\right|}{\left|q_{x}^{\prime}-p_{x}^{\prime}\right|} l^{\prime}=\left|q_{y}^{\prime}-p_{y}^{\prime}\right| \leq \frac{1}{32} .
$$

Moreover, for $\varepsilon^{\prime}=\tau l^{\prime}, \lambda=\frac{s^{\prime}}{s}$ we get $x_{ \pm}^{\prime}=x_{0}^{\prime} \pm \varepsilon^{\prime}, y_{ \pm}^{\prime}=y_{0}^{\prime} \pm m \varepsilon^{\prime}$ and $\varepsilon^{\prime}=\lambda \varepsilon$ as before and again we get the constant

$$
\begin{aligned}
C= & 4(1-\lambda)^{2}-8\left(y_{0}-y_{0}^{\prime}\right) D \\
+ & \frac{8\left(x_{0}-x_{0}^{\prime}\right)\left(y_{0}-y_{0}^{\prime}\right)(1-\lambda)\left(\lambda m+2 D x_{0}\right)-4\left(x_{0}-x_{0}^{\prime}\right)^{2}(1-\lambda)^{2}}{\left(x_{0}-x_{0}^{\prime}\right)^{2}+\left(y_{0}-y_{0}^{\prime}\right)^{2}} .
\end{aligned}
$$

Now, we estimate

$$
\begin{aligned}
C l^{2}\left\|p_{0}-p_{0}^{\prime}\right\|_{2}^{2}= & 4\left(l-l^{\prime}\right)^{2}\left(y_{0}-y_{0}^{\prime}\right)^{2} \\
& -8\left(y_{0}-y_{0}^{\prime}\right) D l^{2}\left(\left(x_{0}-x_{0}^{\prime}\right)^{2}+\left(y_{0}-y_{0}^{\prime}\right)^{2}\right) \\
& +8\left(x_{0}-x_{0}^{\prime}\right)\left(y_{0}-y_{0}^{\prime}\right)\left(l-l^{\prime}\right)\left(m l^{\prime}+2 D x_{0} l\right) \\
\geq & \left(4-576 \delta^{2}-\frac{1}{8}-96 \delta\right)\left(l-l^{\prime}\right)^{2}\left(y_{0}-y_{0}^{\prime}\right)^{2} \\
= & \left(\frac{31}{8}-96 \delta-576 \delta^{2}\right)\left(l-l^{\prime}\right)^{2}\left(y_{0}-y_{0}^{\prime}\right)^{2}>0
\end{aligned}
$$

for $\delta<0.033$.

(b) If $|m|>1$, we have $l \in[4,6]$ and

$$
l^{\prime}=\frac{\sqrt{2}}{2} \sqrt{\left(q_{x}^{\prime}-p_{x}^{\prime}\right)^{2}+\left(q_{y}^{\prime}-p_{y}^{\prime}\right)^{2}} \leq\left|q_{y}^{\prime}-p_{y}^{\prime}\right| \leq \frac{1}{32} .
$$

Furthermore, let $\varepsilon_{x}^{\prime}=x_{+}-x_{0}$ and $\varepsilon_{y}^{\prime}=y_{+}-y_{0}$. Then we have $\varepsilon_{y}^{\prime}=m \varepsilon_{x}^{\prime}$ and

$$
2\left(\tau l^{\prime}\right)^{2}=\varepsilon_{x}^{\prime 2}+\left(m \varepsilon_{x}^{\prime}\right)^{2}=\left(1+m^{2}\right) \varepsilon_{x}^{\prime 2} .
$$

Thus we get $\varepsilon_{x}^{\prime}=\lambda_{x} \varepsilon$ for $\lambda_{x}:=\frac{l^{\prime}}{l} \frac{\sqrt{2}}{\sqrt{1+m^{2}}}, \varepsilon_{y}^{\prime}=\lambda_{y} \varepsilon$ for $\lambda_{y}:=m \lambda_{x}=\frac{l^{\prime}}{l} \frac{\sqrt{2} m}{\sqrt{1+m^{2}}}$, $x_{ \pm}^{\prime}=x_{0}^{\prime} \pm \varepsilon_{x}^{\prime}$ and $y_{ \pm}^{\prime}=y_{0}^{\prime} \pm \varepsilon_{y}^{\prime}$. 
We proceed again as before and get the constant

$$
\begin{aligned}
C= & 4\left(1-\lambda_{x}\right)^{2}-8\left(y_{0}-y_{0}^{\prime}\right) D \\
+ & \frac{8\left(x_{0}-x_{0}^{\prime}\right)\left(y_{0}-y_{0}^{\prime}\right)\left(1-\lambda_{x}\right)\left(\lambda_{y}+2 D x_{0}\right)-4\left(x_{0}-x_{0}^{\prime}\right)^{2}\left(1-\lambda_{x}\right)^{2}}{\left(x_{0}-x_{0}^{\prime}\right)^{2}+\left(y_{0}-y_{0}^{\prime}\right)^{2}},
\end{aligned}
$$

with

$$
\begin{aligned}
D & =\delta(l-4) \leq \delta\left(l-l^{\prime}\right) \leq 6 \delta\left(1-\lambda_{x}\right), \\
y_{0}-y_{0}^{\prime} & \leq D\left(1-x_{0}^{2}\right) \leq D \leq 6 \delta\left(1-\lambda_{x}\right), \\
\lambda_{y} & =\frac{l^{\prime}}{l} \frac{\sqrt{2}}{\sqrt{\frac{1}{m^{2}}+1}} \leq \frac{\sqrt{2}}{128} \leq \frac{1}{64}\left(1-\lambda_{x}\right) .
\end{aligned}
$$

Now, we estimate

$$
\begin{aligned}
C\left\|p_{0}-p_{0}^{\prime}\right\|_{2}^{2}= & 4\left(1-\lambda_{x}\right)^{2}\left(y_{0}-y_{0}^{\prime}\right)^{2} \\
& -8\left(y_{0}-y_{0}^{\prime}\right) D\left(\left(x_{0}-x_{0}^{\prime}\right)^{2}+\left(y_{0}-y_{0}^{\prime}\right)^{2}\right) \\
& +8\left(x_{0}-x_{0}^{\prime}\right)\left(y_{0}-y_{0}^{\prime}\right)\left(1-\lambda_{x}\right)\left(\lambda_{y}+2 D x_{0}\right) \\
\geq & \left(4-576 \delta^{2}-\frac{1}{64}-96 \delta\right)\left(1-\lambda_{x}\right)^{2}\left(y_{0}-y_{0}^{\prime}\right)^{2} \\
= & \left(\frac{255}{64}-96 \delta-576 \delta^{2}\right)\left(1-\lambda_{x}\right)^{2}\left(y_{0}-y_{0}^{\prime}\right)^{2}>0
\end{aligned}
$$

for $\delta<0.034$. Hence this is again true for $\delta \leq \frac{1}{64}$.

Observe that for $m \rightarrow+\infty$ we get $\lambda_{x}=0$ and $\lambda_{y}=\sqrt{2} \frac{l^{\prime}}{l}$, and the same estimates hold.

Proof of Proposition 2.5. The geodesic bicombing $\tilde{\sigma}^{\delta}$ is non-consistent and nonreversible, as observed before.

For convexity, the same arguments as in the proof of Proposition 2.2 apply. The only new case is $p^{\prime} \in X_{+}$and $q^{\prime} \in X_{-}$. With the notions from above with $x_{ \pm}^{\prime}=x_{0}^{\prime} \mp \varepsilon^{\prime}$ for $\varepsilon^{\prime}=\tau l^{\prime}$ and $\lambda=\frac{l^{\prime}}{l}$ we obtain the constant

$$
\begin{aligned}
C= & 4(1+\lambda)^{2}-8 y_{0} D \\
+ & \frac{16\left(x_{0}-x_{0}^{\prime}\right) y_{0}(1+\lambda) x_{0} D-4\left(x_{0}-x_{0}^{\prime}\right)^{2}(1+\lambda)^{2}}{\left(x_{0}-x_{0}^{\prime}\right)^{2}+y_{0}^{2}} .
\end{aligned}
$$

With the inequalities $D=\delta(l-4) \leq 2 \delta$ and $\left|y_{0}\right| \leq \frac{1}{32}$ we get

$$
\begin{aligned}
C\left\|p_{0}-p_{0}^{\prime}\right\|_{2}^{2}= & 4(1+\lambda)^{2} y_{0}^{2} \\
& -8 y_{0} D\left(\left(x_{0}-x_{0}^{\prime}\right)^{2}+y_{0}^{2}\right) \\
& +16\left(x_{0}-x_{0}^{\prime}\right) y_{0}(1+\lambda) x_{0} D \\
\geq & (4-\delta-32 \delta)(1+\lambda)^{2} y_{0}^{2} \\
\geq & (4-33 \delta)(1+\lambda)^{2} y_{0}^{2}>0
\end{aligned}
$$

for $\delta<\frac{4}{33}$, hence for all $\delta \leq \frac{1}{64}$. 
Acknowledgments. We would like to thank Urs Lang for introducing us to conical geodesic bicombings and for his helpful remarks and guidance. We are also thankful for helpful suggestions of the anonymous referee. The authors gratefully acknowledge support from the Swiss National Science Foundation.

\section{References}

[AB06] C. Aliprantis and K. Border. Infinite dimensional analysis: a hitchhiker's guide. Springer, 2006.

[Bas17] G. Basso. Fixed point theorems for metric spaces with a conical geodesic bicombing. Ergodic Theory and Dynamical Systems, pages 1-16, 2017.

[BP87] H. Busemann and B. Phadke. Spaces with distinguished geodesics, volume 108. Marcel Dekker Inc, 1987.

[Des16] Dominic Descombes. Asymptotic rank of spaces with bicombings. Math. Z., 284(3-4):947960, 2016.

[DL15] D. Descombes and U. Lang. Convex geodesic bicombings and hyperbolicity. Geometriae Dedicata, 177(1):367-384, 2015.

[DL16] D. Descombes and U. Lang. Flats in spaces with convex geodesic bicombings. Analysis and Geometry in Metric Spaces, 4(1):68-84, 2016.

[Dre84] A. W. M. Dress. Trees, tight extensions of metric spaces, and the cohomological dimension of certain groups: a note on combinatorial properties of metric spaces. Advances in Mathematics, 53(3):321-402, 1984.

[GM81] S. Gähler and G. Murphy. A metric characterization of normed linear spaces. Mathematische Nachrichten, 102(1):297-309, 1981.

[Goo50] D. B. Goodner. Projections in normed linear spaces. Transactions of the American Mathematical Society, 69(1):89-108, 1950.

[Kel52] J. L. Kelley. Banach spaces with the extension property. Transactions of the American Mathematical Society, 72(2):323-326, 1952

[Kel16] M. Kell. Sectional curvature-type conditions on finsler-like metric spaces. arXiv preprint arXiv:1601.03363, 2016.

[Lan13] U. Lang. Injective hulls of certain discrete metric spaces and groups. Journal of Topology and Analysis, 5(03):297-331, 2013.

[LY10] Y.-C. Li and C.-C. Yeh. Some characterizations of convex functions. Computers 85 mathematics with applications, 59(1):327-337, 2010.

[Mie16] B. Miesch. The Cartan-Hadamard Theorem for Metric Spaces with Local Geodesic Bicombings. arXiv preprint arXiv:1509.07001, 2016.

[RS90] S. Reich and I. Shafrir. Nonexpansive iterations in hyperbolic spaces. Nonlinear Analysis: Theory, Methods and Applications, 15(6):537-558, 1990.

[Tak70] W. Takahashi. A convexity in metric space and nonexpansive mappings. I. Kodai Mathematical Seminar Reports, 22(2):142-149, 1970.

Mathematik Departement, ETH Zürich, Rämistrasse 101, 8092 Zürich, SchweiZ

E-mail adress: giuliano.basso@math.ethz.ch

E-mail adress: benjamin.miesch@math.ethz.ch 